\title{
ИММУНОТРОПНЫЕ ЭФФЕКТЫ ПОЛИОКСИДОНИЯ В КОМПЛЕКСНОМ ЛЕЧЕНИИ БОЛЬНЫХ С ПАРАРЕКТАЛЬНЫМИ СВИЩАМИ
}

\author{
(С Лазаренко В.А. ${ }^{1}$, Заикин Е.Ю. ${ }^{l}$, Калуцкий П.В. ${ }^{2}$, Калуцкий А.П. ${ }^{1}$ \\ ${ }^{1}$ Кафедра хирургических болезней ФПО, ${ }^{2}$ кафедра микробиологии, вирусологии, иммунологии \\ Курского государственного медицинского университета, Курск \\ E-mail:pvk62@mail.ru
}

Исследовано влияние традиционной терапии, дополненной полиоксидонием, на состояние клеточного иммунитета и цитокиновый статус крови больных с параректальными свищами. Установлено, что развитие параректальных свищей сопровождается формированием выраженного дисбаланса субпопуляций лимфоцитов и цитокинов. Анализ эффективности схемы лечения у пациентов с хроническим парапроктитом по сумме показателей, отличных от уровня нормы, показал, что назначение дополнительно к стандартной схеме лечения полиоксидония позволило нормализовать $11,5 \%$ показателей, корригировать 27,0\% показателей, тогда как 34,5\% показателей оставались после проведенного лечения без изменения. Таким образом, назначение дополнительно к стандартной схеме терапии полиоксидония хотя и приводит к коррекции ряда нарушенных иммунометаболических показателей, но не позволяет добиться нормализации рабалансированных параметров иммунитета у больных с параректальными свищами, что свидетельствует о недостаточной иммунотропной (иммунокорригирующей) составляющей использованной схемы иммунореабилитации у данной категории пациентов.

Ключевые слова: параректальные свищи, клеточный иммунитет, цитокины, традиционная терапия, полиоксидоний.

\section{IMMUNOTROPIC EFFECTS OF POLYOXIDONIUM IN THE COMPLEX TREATMENT OF PATIENTS WITH PARARECTAL FISTULAS \\ Lazarenko V.A. ${ }^{l}$, Zaikin E.Y. ${ }^{1}$, Kalutsky P.V. ${ }^{2}$, Kalutsky A.P. ${ }^{l}$ \\ ${ }^{1}$ Department of Surgical Diseases of FPE, ${ }^{2}$ Department of Microbiology, Virology, Immunology of Kursk State Medical University, Kursk}

The influence of traditional therapy supplemented with polyoxidonium on the state of cellular immunity and the cytokine status of blood of patients with pararectal fistulas was studied. It was established that the development of pararectal fistula is accompanied by forming a marked imbalance in the subpopulations of lymphocytes and cytokines. Analysis of the efficacy of the treatment regimen in patients with chronic paraproctitis in terms of the number of indicators different from the normal level showed that the administration of polyoxidonium in addition to the standard treatment regimen allowed to normalize $11.5 \%$ of the indicators, $27.0 \%$ of the parameters, while $34.5 \%$ had no change after the treatment. Thus, the administration of polyoxidonium in addition to the standard therapy, despite having the correction of a number of the immunometabolic parameters, does not permit to normalize the disturbed parameters of immunity in patients with pararectal fistulas indicating an insufficient immunotropic (immunocorrecting) component of the used immunorehabilitation scheme in this category of patients.

Keywords: adrectal fistula, cellular immunity, cytokines, traditional therapy, polyoxidonium.

Несмотря на большие успехи в создании антимикробных средств, лечение инфекционновоспалительных заболеваний в XXI веке остается непростой и порой достаточно трудной задачей. Основными причинами этого являются проблема антибиотикорезистентности и широкая распространенность иммунодефицитных состояний, которые могут быть как фоном при развитии острой инфекции, так и следствием длительно протекающей хронической инфекции. Эти факторы затрудняют, а в некоторых случаях делают невозможным достижение ремиссии с использованием стандартных средств - хирургического вмешательства и/или антимикробной фармакотерапии [9]. К одному из таких хирургических заболеваний относится хронический парапроктит, включающий в себя различные формы параректальных свищей.
Течение и исход заболевания в значительной степени зависит от состояния иммунологической реактивности организма [1]. Поскольку параректальные свищи, как и все гнойно-воспалительные заболевания, сопровождаются нарушением иммунного статуса, то одним из адекватных способов комплексного лечения представляется иммунотерапия препаратами системного и локального действия [7, 8]. Недостаточная эффективность этиотропной терапии заболевания делает актуальной оптимизацию методов патогенетической и, в частности, иммунотропной терапии нарушений в системе иммунитета [2].

Целью настоящего исследования явилось изучение иммунологической эффективности у больных со свищевой формой хронического парапроктита традиционной терапии, дополненной полиоксидонием. 


\section{МАТЕРИАЛЫ И МЕТОДЫ ИССЛЕДОВАНИЯ}

В исследовании на основе добровольного согласия приняли участие 60 больных с подтвержденным диагнозом хронического парапроктита в возрасте от 31 года до 60 лет, поступивших для лечения в колопроктологическое отделение ОГБУЗ «Белгородская областная клиническая больница святителя Иоасафа». В зависимости от схемы терапии все пациенты были разделены на 2 группы. Пациенты первой группы (n=28) получали стандартное лечение, включавшее хирургическое вмешательство. Вид вмешательства определялся в зависимости от вида параректального свища, наличия гнойных затеков, рубцовых изменений в анальном канале. В послеоперационном периоде пациенты получали противовоспалительную терапию, анальгетики, производилось регулярное выполнение перевязок, заключающихся в очищении ран растворами антисептиков и нанесении на раневую поверхность мазевых основ на водорастворимой основе. Пациенты второй группы $(\mathrm{n}=32)$ в дополнение к стандартному лечению получали полиоксидоний в дозе 6 мг внутримышечно 1 раз в сутки через день, общий курс 5 инъекций.

У всех больных при поступлении производился забор венозной крови, в которой методом проточной цитометрии на аппарате Beckman Culter Epecs XL c моноклональными антителами оценивали содержание клеток с фенотипами $\mathrm{CD}^{+} \mathrm{CD}^{-} 9^{-}, \mathrm{CD}^{+} \mathrm{CD}^{+}, \mathrm{CD}^{+} \mathrm{CD}^{+}, \mathrm{CD}^{+}{ }^{+} \mathrm{HLA}-$ $\mathrm{DR}^{+}, \mathrm{CD}^{+}{ }^{+} \mathrm{CD} 16^{+} 56^{+}, \mathrm{CD}^{-} \mathrm{CD} 16^{+} 56^{+}$. Кроме того, в сыворотке крови методом твердофазного иммуноферментного анализа с помощью набора реагентов ВЕКТОР-БЕСТ (ООО «ВЕКТОР-БЕСТ», г. Новосибирск) оценивали уровни следующих

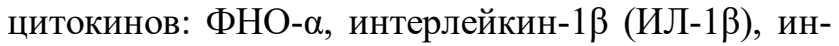
терлейкин-2 (ИЛ-2), интерлейкин-8 (ИЛ-8), интерлейкин-10 (ИЛ-10), интерферон- $\gamma$ (ИНФ- $\gamma)$ и рецепторный антагонист интерлейкина-1 (РАИЛ1). На десятые сутки лечения проводился повторный забор 10 мл венозной крови у каждого пациента с целью оценки изменений иммунного статуса. В качестве контроля использовали результаты исследований показателей у 20 здоровых добровольцев.

В работе со здоровыми людьми и больными хроническим парапроктитом соблюдались этические принципы, предъявляемые Хельсинкской декларацией Всемирной медицинской ассоциации [11] и «Правилами клинической практики в Российской Федерации», утвержденными Приказом Минздрава РФ от 19.06.2003 № 266.
Степень иммунных расстройств (СИР) для иммунологических показателей рассчитывали по формуле [6]:

$$
\left(\frac{\text { показатель конкретного больного }}{\text { показатель, принятый за норму }}-1\right) \times 100 \%
$$

По всем показателям рассчитывали коэффициент диагностической ценности, с помощью которого формулу иммунных расстройств иммунной системы (ФРИС) путем выбора изо всех изученных параметров трех ведущих, наиболее отличающихся от уровня нормы [6].

Статистическая обработка полученных данных проводилась с помощью пакетов прикладных программ «Excel» и «Statistica 8.0». При проверке исследуемых групп на нормальность распределения с использованием критериев Шапиро-Уилка и $\chi^{2}$ выявлено отклонение от нормального закона распределения. Поэтому для сравнения иммунологических показателей контрольной и изучаемых групп использовался непараметрический вариант критерия Ньюмена-Кейлса [3, 4]. Статистически значимыми считали различия с $\mathrm{p}<0,05$.

\section{РЕЗУЛЬТАТЫ ИССЛЕДОВАНИЯ И ИХ ОБСУЖДЕНИЕ}

В ходе проведенного обследования на базе колопроктологического отделения ОГБУЗ «Белгородская областная клиническая больница Святителя Иоасафа» 60 больных с хроническим парапроктитом были установлены достаточно выраженные изменения иммунного статуса на системном уровне и ряда метаболических показателей.

У больных с параректальными свищами имели место значительные изменения со стороны $73,0 \%$ изученных показателей, характеризующих состояние иммунной системы организма: повышение по сравнению с данными здоровых лиц абсолютного количества лимфоцитов, несущих маркеры $\mathrm{CD}^{+} \mathrm{CD}^{+}, \mathrm{CD}^{-} \mathrm{CD}^{-} 6^{+} 56^{+}$и $\mathrm{CD}^{+}{ }^{+} \mathrm{HLA}-$ $\mathrm{DR}^{+}$, а также относительного содержания клеток с фенотипом $\mathrm{CD}^{+}{ }^{+} \mathrm{CD} 19^{-}, \mathrm{CD}^{+}{ }^{+} \mathrm{CD} 8^{+}, \mathrm{CD}^{+} \mathrm{HLA}-$ $\mathrm{DR}^{+}, \mathrm{CD}^{+} \mathrm{CD}^{1} 6^{+} 56^{+}$и $\mathrm{CD}^{-} \mathrm{CD} 16^{+} 56^{+}$(рис. 1). Вместе с тем абсолютное число лимфоцитов $\mathrm{CD}^{+}{ }^{+} \mathrm{CD} 19^{-}$и $\mathrm{CD}^{+} \mathrm{CD} 16^{+} 56^{+}$было ниже значений группы контроля.

Что касается уровня провоспалительных цитокинов, то необходимо отметить их различную реакцию на развитие патологического процесса (рис. 2). Наиболее выраженной она была со стороны ИЛ-8, поэтому именно данный показателей вошел в ФРИС и занял первое место, тогда как на втором месте оказался уровень ИЛ- $1 \beta$ и ФНО- $\alpha$. СИР всех показателе, входящих в ФРИС, имела III степень, т.е. превышала $66 \%$ предел от уровня нормы (табл. 1). 


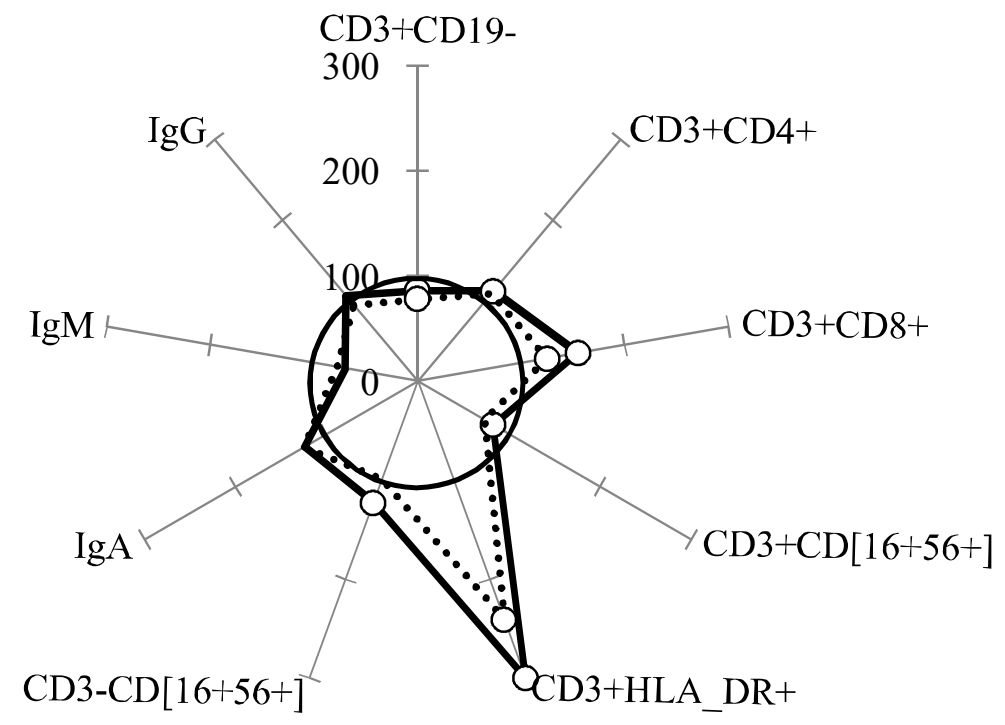

Рис. 1. Реакция клеточного звена иммунитета и иммуноглобулинов больных с хроническими парапроктитами на стандартную терапию, дополненную полиоксидонием.

Примечание: сплошная линия - показатели у больных до лечения; пунктирная линия - показатели у больных после лечения; o - p $<0,05$ по отношению к показателям контрольной группы.

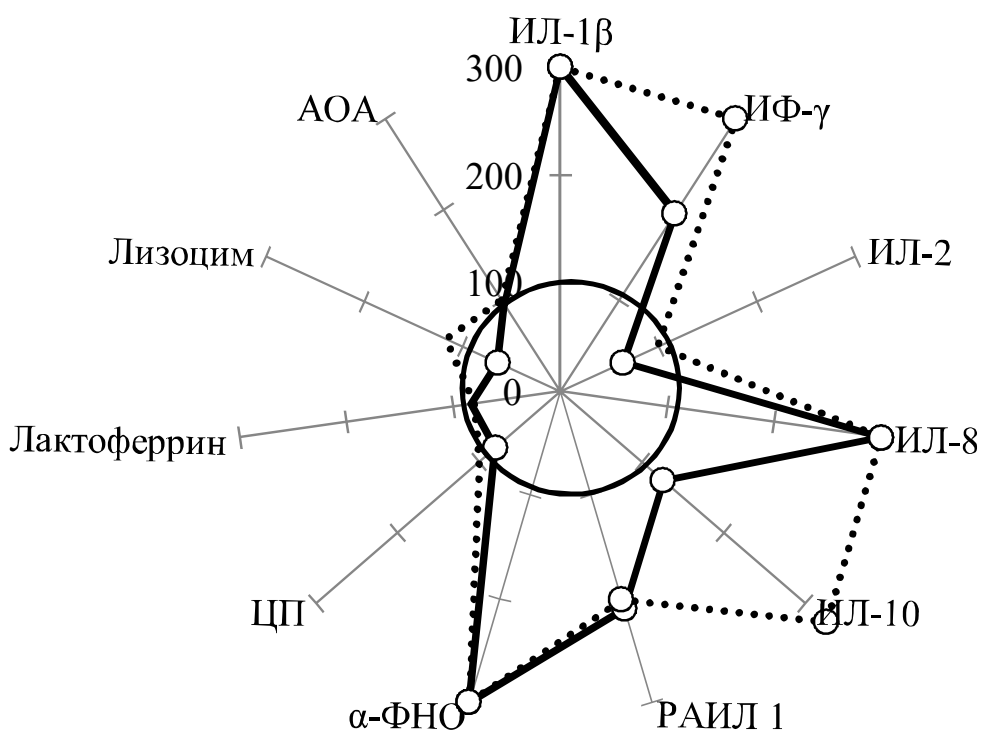

Рис. 2. Уровни цитокинов, лизоцима, лактоферрина, церулоплазмина и антиоксидантной активности у больных с хроническим парапроктитом на фоне стандартной терапии, дополненной полиоксидонием.

Примечание: сплошная линия - показатели у больных до лечения; пунктирная линия - показатели у больных после лечения; o - p $<0,05$ по отношению к показателям контрольной группы

Таблица 1

Рейтинговый алгоритм иммунологических показателей у больных, получавших полиоксидоний

\begin{tabular}{|c|c|c|c|c|c|c|}
\hline \multirow{2}{*}{ Группы больных } & \multirow{2}{*}{\multicolumn{3}{|c|}{ ФРИС }} & \multicolumn{3}{|c|}{ СИР } \\
\hline & & & & I & II & III \\
\hline До лечения & ${\text { ИЛ }-8_{3}^{+}}^{+}$ & ИЛ- $1 \beta_{3}{ }^{+}$ & $\Phi \mathrm{HO}_{3}{ }^{+}$ & 2 & 5 & 8 \\
\hline После стандартного лечения & ИЛ- $8_{3}^{+}$ & ИЛ- $1 \beta_{3}{ }^{+}$ & $\Phi \mathrm{HO}_{3}{ }^{+}$ & 1 & 4 & 8 \\
\hline После стандартного лечения + полиоксидоний & ИЛ $-8_{3}^{+}$ & ИЛ- $1 \beta_{3}{ }^{+}$ & $\Phi \mathrm{HO}_{3}{ }^{+}$ & - & 1 & 6 \\
\hline
\end{tabular}


В группах больных парапроктитом до проведения лечения отмечается увеличение относительно показателей группы контроля концентрации лизоцима, тогда как концентрация иммуноглобулинов $\mathrm{A}, \mathrm{G}$ и М в сыворотке крови и церуллоплазмина достоверно не отличалась от показателей контрольной группы.

Полученные результаты свидетельствуют о разнонаправленной реакции со стороны цитокинового звена иммунитета у больных с параректальными свищами. При этом неодинаковый характер реакции на развитие патологического процесса регистрировался не только среди цитокинов различных групп, но и внутри группы провоспалительных цитокинов. Последнее может быть связано с особенностями биологических характеристик исследованных представителей данной группы цитокинов.

Неадекватная ответная реакция противовоспалительных цитокинов может служить основой для неблагоприятного развития патологического процесса, поскольку дисбаланс в выработке цитокинов может нарушить существующие в локальной системе взаимосвязи, что в конечном итоге приводит к патологии регенерации.

Как показали проведенные нами исследования [5], стандартная схема лечения у пациентов с хроническим параректальными свищами не нормализует, т.е. не восстанавливает до контрольных значений, ни одного из изученных показателей иммунного статуса, не оказывает влияния на представительность ФРИС у данной категории пациентов. Все это позволяет сделать вывод о том, что использование стандартной терапии у пациентов с хроническим парапроктитом не позволяет существенно снизить количество показателей иммунного статуса со второй и третьей СИР, тем самым диктуя необходимость поиска и апробирования в клинических исследованиях новых и эффективных способов фармакологической иммунореабилитации с использованием иммуностимуляторов.

В качестве препарата для коррекции иммунометаболического статуса был выбран хорошо изученный препарат из группы иммуностимуляторов - полиоксидоний (сополимер $\mathrm{N}$-оксида 1,4-этиленпиперазина и (N-карбоксиметил)1,4-этиленпиперазиний бромида; ООО «НПО Петровакс Фарм»; Россия). Полиоксидоний был выбран из-за его важного для хронической бактериальной инфекции свойства - действовать на все звенья фагоцитарного процесса. Полиоксидоний активирует миграцию фагоцитов, усиливает клиренс чужеродных частиц из кровотока, повышает поглотительную и бактерицидную активность фагоцитов. При оценке последнего параметра с помощью проточной цитометрии и «двойной метки» было установлено, что полиоксидоний в разы усиливает способность фагоцитов периферической крови нормальных доноров убивать $\mathrm{S}$. aureus и это усиление носит дозозависимый характер [10].

Как показали проведенные исследования, использование полиоксидония у пациентов с хроническим парапроктитом не оказывает влияния в крови на измененный в абсолютных и относительных значениях уровень представительности $\mathrm{CD}^{+}{ }^{+} \mathrm{CD} 19^{-}$и $\mathrm{CD}^{+} \mathrm{CD} 16^{+} 56^{+}$лимфоцитов, относительный уровень $\mathrm{CD}^{-} \mathrm{CD} 16^{+} 56^{+}$и $\mathrm{CD}^{+} \mathrm{HLA}$ $\mathrm{DR}^{+}$клеток и абсолютные значения количества $\mathrm{CD}^{+} \mathrm{CD}^{+}$лимфоцитов (рис. 1). В то же время применение препарата снижает, но не до уровня нормы в абсолютных значениях представительность $\mathrm{CD} 3^{+} \mathrm{CD} 4^{+}$клеток и абсолютные значения $\mathrm{CD}^{+}{ }^{+} \mathrm{HLA}^{-\mathrm{DR}^{+}}$и $\mathrm{CD} 3^{-} \mathrm{CD} 16^{+} 56^{+}$лейкоцитов. Назначение дополнительно к стандартной схеме лечения полиоксидония нормализует из изученных показателей клеточного звена иммунитета только относительный уровень $\mathrm{CD}^{+} \mathrm{CD}^{+}$лейкоцитов.

Использование иммуностимулятора у пациентов не оказывает влияния на измененную концентрацию в плазме крови РАИЛ 1, ФНО- $\alpha$ и лактоферрина, повышает концентрацию в плазме крови ИЛ-1 $\beta$, ИФ- $\gamma$, ИЛ-8 и ИЛ-10 (рис. 2). Однако применение полиоксидония дополнительно к стандартной схеме лечения снижает у больных до уровня контрольной группы концентрацию в плазме крови ИЛ-2.

Анализ эффективности использованной схемы лечения у пациентов с хроническим парапроктитом по сумме показателей, отличных от уровня нормы, показал, что назначение дополнительно к стандартной схеме лечения полиоксидония позволило нормализовать $11,5 \%$ показателей, корригировать $27,0 \%$ показателей, тогда как $34,5 \%$ показателей остались после проведенного лечения без изменения. При анализе степени иммунных расстройств у пациентов с хроническим парапроктитом установлено, что если до лечения у больных показателей с первой СИР было 2, со второй -5 , с третьей -8 , т.е. 15 показателей требуют дополнительной фармакологической коррекции, то на фоне проведенной стандартной схемы лечения пациентов с хроническим парапроктитом первую СИР имел 1 показатель, вторую -4 показателя и третью -8 показателей (табл. 1). Применение стандартной схемы лечения в сочетании с полиоксидонием позволило снизить количество показателей, требующих коррекции, до 7 (1 со второй СИР, 6 с третьей СИР).

Таким образом, назначение дополнительно к стандартной схеме терапии полиоксидония хотя и приводит к коррекции ряда нарушенных имму- 
нометаболических показателей, но не позволяет добиться нормализации рабалансированных параметров иммунитета у больных с параректальными свищами, что свидетельствует о недостаточной иммунотропной (иммунокорригирующей) составляющей использованной схемы иммунореабилитации у данной категории пациентов.

\section{ЛИТЕРАТУРА}

1. Белобородова Н.В., Бачинская Е.Н. Иммунологические аспекты послеоперационного сепсиса // Анестезиология и реаниматология. - 2000. № 1. - C. 59-66.

2. Белохвостикова Ю.С. Цитокинотерапия в лечении гнойной хирургической инфекции // Аллергология и иммунология. - 2005. - Т. 6, № 2. - С. 173.

3. Глани С. Медико-биологическая статистика. Пер. с англ. - М. : Практика, 1998. - 459 с.

4. Гублер Е.В., Генкин А.А. Применение непараметрических критериев статистики в медикобиологических исследованиях. - Л. : Медицина, 1973. - 2-е издание. - 141 с.

5. Заикин Е.Ю., Лазаренко В.А., Калуцчкий П.В., Калуикий А.П. Иммунологическая эффективность традиционной терапии свищевой формы хронического парапроктита // Курский научно- практический вестник «Человек и его здоровье». 2017. - № 2. - С. 71-73.

6. Земсков А.М., Земсков В.М. Дополнительные аспекты оценки иммунного статуса // Клиническая и лабораторная диагностика. - 1994. - № 5. C. 91-98.

7. Караулов А.В. Клиническая иммунология и аллергология. - М., 2002. - 656 с.

8. Кочелев П.И., Резников К.М., Гридин А.А. Лечение гнойных ран с применением анолита и католита // Системный анализ и управление в биомедицинских системах. - 2005. - Т. 4, № 1. - С. 54-56.

9. Пащенков М.В., Будихина А.С., Голубева Н.М., Алхазова Б.И., Львов В.Л., Ступин В.А., Привидениев А.И., Трушин С.Н., Селиверстов Д.В., Огорельцев А.Ю., Пинегин Б.В., Хаитов Р.М. Результаты II/III фазы клинических испытаний иммуномодулятора «Полимурамил» при гнойной хирургической инфекции // Иммунология. - 2012. - Т. 33, № 4. - С. 199-203.

10. Пинегин Б.В., Некрасов А.В., Хаитов Р.М. Иммуномодулятор Полиоксидоний: механизмы действия и аспекты клинического применения // Цитокины и воспаление. - 2004. - Т. 3, № 3. C. 41-47.

11. World Medical Association Declaration of Helsinki / Bulletin of the World Health Organization. - 2001. Vol. 79, N 4. - P. 373-374. 